\title{
Long-term radiation-induced optical darkening effects in chalcogenide glasses
}

\author{
T.S. Kavetskyy \\ Drohobych Ivan Franko State Pedagogical University, \\ 24, I. Franko str., 82100 Drohobych, Ukraine \\ The John Paul II Catholic University of Lublin, \\ 14, Al. Racławickie, 20-950 Lublin, Poland
}

\begin{abstract}
In this work, it is reported that the $\gamma$-irradiated (2.41 MGy accumulated dose) glasses $\mathrm{As}_{2} \mathrm{~S}_{3}$ ( $\sim 2 \mathrm{~mm}$ thick) and $\mathrm{Ge}_{15.8} \mathrm{As}_{21} \mathrm{~S}_{63.2}$ ( 1 mm thick), both measured $\sim 10$ years after $\gamma$-irradiation, exhibit radiation-induced optical darkening effect (i.e., long-wave shift of fundamental optical absorption edge). In the case of $\mathrm{As}_{2} \mathrm{~S}_{3}$ glass, the observed longterm radiation-induced optical darkening effect is well comparable with that reported in literature for $\gamma$-irradiated ( $\sim 3 \mathrm{MGy}$ accumulated dose) glass $\mathrm{As}_{2} \mathrm{~S}_{3}$ (1.5 mm thick), measured directly after $\gamma$-irradiation. In view of practical application, this finding demonstrates the possibilities for development of innovative chalcogenide glass based long-term dosimeter systems with stable and controlled parameters. A possible general criterion for mechanisms of long-term radiation-induced structural changes in chalcogenide glasses has been also considered.
\end{abstract}

Keywords: chalcogenide glasses, optical properties, radiation modification.

Manuscript received 20.06.16; revised version received 05.09.16; accepted for publication 16.11.16; published online 05.12.16.

\section{Introduction}

Radiation-induced optical effects (RIOEs) caused by ${ }^{60} \mathrm{Co} \gamma$-irradiation in chalcogenide glasses (ChGs) are well-known to be used for dosimetric applications [1, 2]. RIOEs could be also considered as the main control parameter of radiation sensitivity of ChGs. It has been established on the example of Ge-As-S [3] and Ge-Sb-S [4] systems that the total RIOEs consist of two components: the dynamic component that relaxes for 2-3 months after $\gamma$-irradiation and static one that remains stable for a long period of time after $\gamma$-irradiation.

Recently, analyzing the radiation-induced effects in chalcogenide glasses the authors [5] made assumption that a long-term period (more than 7 years) after ${ }^{60} \mathrm{Co} \gamma$ - irradiation does not give an argument for observation of radiation-induced effects caused by timing relaxation of post-radiation changes or dynamic component of RIOE. But, as mentioned above, it has been experimentally established $[3,4]$ that the RIOE's dynamic component is only 2 to 3 months and RIOE's static component takes place for a long period after $\gamma$-irradiation. However, the question "how long RIOE's static component would be existed?" was not examined yet.

In this Letter, the results of optical transmission measurements of $\mathrm{As}_{2} \mathrm{~S}_{3}\left(\sim 2 \mathrm{~mm}\right.$ thick) and $\mathrm{Ge}_{15.8} \mathrm{As}_{21} \mathrm{~S}_{63.2}$ ( $\sim \mathrm{mm}$ thick) glasses in unirradiated and $\gamma$-irradiated (2.41 MGy accumulated dose) states in the range of fundamental optical absorption edge are reported for the samples measured $\sim 10$ years after $\gamma$-irradiation. 
The investigated samples of $\mathrm{As}_{2} \mathrm{~S}_{3}$ and $\mathrm{Ge}_{15.8} \mathrm{As}_{21} \mathrm{~S}_{63.2}$ compositions were prepared from elements of $99.9999 \%$ purity in evacuated silica ampoules using the standard melt-quenching procedure [6], polished to a high optical quality and annealed at about $20 \ldots 30 \mathrm{~K}$ below the corresponding glass transition temperature before their radiation treatment. Both samples were subjected to radiation treatment with ${ }^{60} \mathrm{Co}$ $\gamma$-quanta with the average energy $E=1.25 \mathrm{MeV}$ and accumulated dose $\Phi=2.41 \mathrm{MGy}$, details of which are described elsewhere [7-9]. Optical transmission spectra of the samples were measured in the visible spectral range at room temperature using an Evolution $220 \mathrm{UV}$ visible spectrophotometer.

Fig. 1 shows the optical transmission spectra, $\tau(\lambda)$, of $\mathrm{g}-\mathrm{As}_{2} \mathrm{~S}_{3}$ and $\mathrm{g}-\mathrm{Ge}_{15.8} \mathrm{As}_{21} \mathrm{~S}_{63.2}$ samples in the unirradiated and $\gamma$-irradiated states, measured $\sim 10$ years after $\gamma$-irradiation. Clearly, the long-wave shift of fundamental optical absorption edge is detected, signifying the existence of long-term radiation-induced optical darkening effect. The decrease in the sample's transparency in the saturation region for $\mathrm{g}-\mathrm{As}_{2} \mathrm{~S}_{3}$ is due to the radiation-induced oxidation processes occurring on the sample surface [9]. The observed long-term radiationinduced optical darkening effect is found to be practically the same as that reported for $\gamma$-irradiated ( 3 MGy

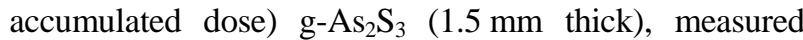
directly after $\gamma$-irradiation [10]. To quantify this similarity, as an example, one may compare $\Delta \tau \sim 15 \%$ at $\lambda=675 \mathrm{~nm}$ in this study (see Fig. 1) with $\Delta \tau \sim 20 \%$ at $\lambda=675 \mathrm{~nm}$ in the work [10] (see Fig. 1, curves 1 and 2). Taking into account the dynamic RIOE component on the level of $\sim 5 \%[3,4]$, it means that the RIOE's static component may exist for a long period, perhaps more than 10 years. It may also indicate that a backward measuring chronology mentioned by the authors [10], it is not necessary to be performed, being although useful to make in situ experiment, as the radiation-induced optical darkening effects in chalcogenide glasses are stable in time, and corresponding radiation-induced structural changes can be correctly investigated with a long-time interval. In this respect, the long-term radiation-induced structural changes examined in the works [2, 11-14] are very important in view of practical applications of chalcogenide glasses. In particular, the long-term radiation-induced effects demonstrate the possibilities for development of innovative chalcogenide glass based longterm dosimeter systems with stable and controlled parameters.

In spite of the long-term radiation-induced optical darkening effects detected in this study for both investigated $g-\mathrm{As}_{2} \mathrm{~S}_{3}$ and $\mathrm{g}-\mathrm{Ge}_{15.8} \mathrm{As}_{21} \mathrm{~S}_{63.2}$, the mechanisms of long-term radiation-induced structural changes in these compounds are found to be different [2, $11,13,14]$. It is concluded from the recent positron annihilation spectroscopy measurements with the Doppler broadening of annihilation line (DBAL) technique [11, 14]. Namely, it has been ascertained using the DBAL data that the defect structures of radiation-modified $\mathrm{g}-\mathrm{As}_{2} \mathrm{~S}_{3}$ and $\mathrm{g}-\mathrm{Ge}_{15.8} \mathrm{As}_{21} \mathrm{~S}_{63.2}$ are different probably as a result of different mechanisms of radiation-induced defect formation. It has been suggested that the non-defective mechanism within the distortion model for $\mathrm{g}-\mathrm{As}_{2} \mathrm{~S}_{3}$ and the defective mechanism within the coordination topological defects (CTDs) model for g- $\mathrm{Ge}_{15.8} \mathrm{As}_{21} \mathrm{~S}_{63.2}$ took place [11, 14], since the latter CTD model has been evidently verified using Raman spectroscopy studies, employing the differential representation of depolarized Raman spectra [13]. The CTD approach used for g- $\mathrm{Ge}_{15.8} \mathrm{As}_{21} \mathrm{~S}_{63.2}$ or $\left(\mathrm{As}_{2} \mathrm{~S}_{3}\right)_{0.4}\left(\mathrm{GeS}_{2}\right)_{0.6}$ alloy with the dominant Ge-S subsystem seems to be valid for major Ge-S based chalcogenides (see, for example, [15-17]).

Therefore, the positron annihilation spectroscopy DBAL method should be reported as a highly sensitive and unique experimental tool to compensate impossibilities (if any) of optical and structural techniques to directly identify the radiation-induced changes in defect structure of glassy materials on the nanoscale level. Moreover, analyzing the DBAL experimental results obtained in the context with XRD and Raman data, some regularity could be observed that may be applied to estimate a possible general criterion for mechanisms of long-term radiation-induced structural changes in ChGs.

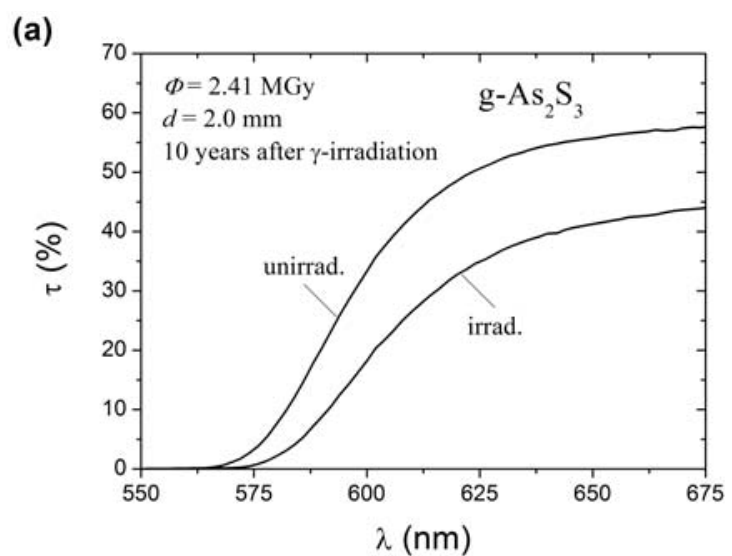

(b)

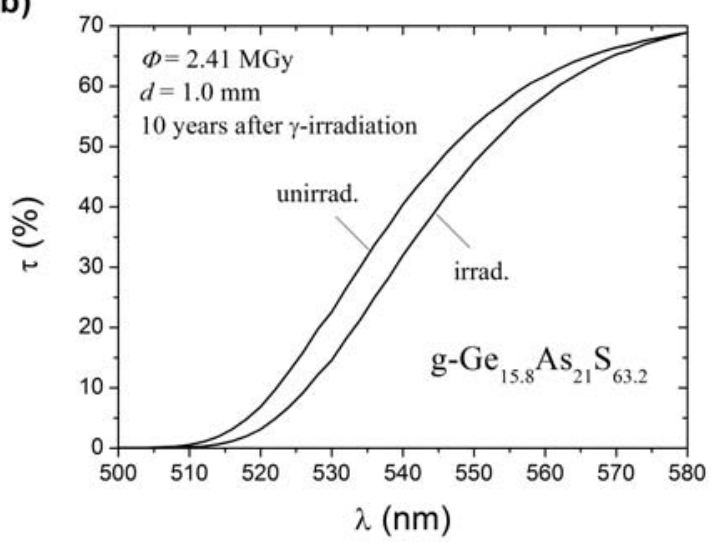

Fig. 1. Optical transmission spectra, $\tau(\lambda)$, of (a) $\mathrm{g}-\mathrm{As}_{2} \mathrm{~S}_{3}(d \cong$ $2.0 \mathrm{~mm}, \Phi=2.41 \mathrm{MGy})$ and (b) $\mathrm{g}^{-} \mathrm{Ge}_{15.8} \mathrm{As}_{21} \mathrm{~S}_{63.2}(d \cong 1.0 \mathrm{~mm}$, $\Phi=2.41$ MGy) samples in unirradiated (unirrad.) and $\gamma$ irradiated (irrad.) states, measured $\sim 10$ years after $\gamma$-irradiation. 
Indeed, on the one hand, the established for As-S based glasses the long-term radiation-induced structural changes in DBAL data [2,11, 14], when the first sharp diffraction peak (FSDP) becomes weaker and broader after $\gamma$-irradiation [2,12], have been interpreted within the non-defective distortion model. Since the FSDP originates as a signature of the medium-range order (MRO) structure within the range $5 . .20 \AA$ in covalent glasses (for review, see [18]), which is commonly accepted by many researchers in literature, the FSDP weakening and broadening means the decrease in the MRO structural correlation length and, consequently, the rise of disordering on the MRO scale. Note, that no changes or possible ordering on the short-range order (SRO) structure within the range 2 to $5 \AA$ [18] should be happened as a result of radiation-induced decreasing the size or void concentration due to relaxation processes within the distortion model [14]. Thus, when the radiation-induced structural changes in ChGs occur towards the disordering on the MRO scale and no changes or ordering on the SRO scale, the non-defective mechanism in the frame of the distortion model and any other models (if exist), resulting in a final structural configuration without charged defects, is dominant. On the other hand, the established for Ge-S based glasses the long-term radiation-induced structural changes in DBAL data $[2,11,14]$, when the FSDP remains practically unchanged [2] or its intensity can be something higher [16] and peak is probably something narrower upon $\gamma$ irradiation, have been interpreted within the defective CTD model, involving atomic rearrangements directly in the first coordination shells (first-nearest-neighbor atomic correlations), that is, on the SRO scale. The unobservable changes in the FSDP and/or the FSDP sharping and narrowing means that no changes or increasing the MRO structural correlation length and, consequently, a stable MRO structure and/or rise of ordering on the MRO scale take place. Thus, when the radiation-induced structural changes in ChGs occur towards the no changes or ordering on the MRO scale and disordering on the SRO scale, the defective mechanism in the frame of the coordination topological defects and any other models (if exist), resulting in a final structural configuration with charged defects, is dominant.

The above mentioned DBAL data versus FSDPXRD data, interpreted within CTD model for g$\mathrm{Ge}_{15.8} \mathrm{As}_{21} \mathrm{~S}_{63.2}$, are also found to be consistent with Raman data, showing clear confirmation for CTD model applied [13]. In view of the above analysis, the degree of disorder, exemplified by the ratio of $I_{\mathrm{bos}} / I_{\mathrm{mol}}$ in Raman spectrum, illustrating the essential increase of the degree of disorder after $\gamma$-irradiation for g- $\mathrm{Ge}_{15.8} \mathrm{As}_{21} \mathrm{~S}_{63.2}$ [13], should be related to the increase in the disorder on the SRO scale in agreement again with CTD approach. Thus, the origin of $I_{\mathrm{bos}} / I_{\mathrm{mol}}$ seems to be related with the degree of disorder of SRO structure in covalent glasses. It is also some signature that the first sharp diffraction peak and the low frequency dynamics (the boson peak) are not related directly to each other on the MRO scale, which is in contrast to assumption made by the authors [19] and in agreement with observations of the authors [20].

Let us summarize the possible general criterion for mechanisms of long-term radiation-induced structural changes in chalcogenide glasses that involves "orderingdisordering" on the nanoscale as follows: when the radiation-induced structural changes in chalcogenide glasses occur towards disordering on the medium-range order scale and no changes or ordering on the shortrange order scale, the non-defective mechanism, resulting in a final structural configuration without charged defects, is dominant; and, vice versa, when the radiationinduced structural changes in chalcogenide glasses occur towards no changes or ordering on the medium-range order scale and disordering on the short-range order scale, the defective mechanism, resulting in a final structural configuration with charged defects, is dominant.

In conclusion, the long-term radiation-induced optical darkening effects have been detected for $\mathrm{g}-\mathrm{As}_{2} \mathrm{~S}_{3}$

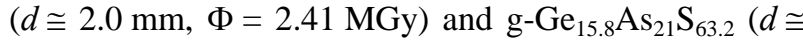
$1.0 \mathrm{~mm}, \Phi=2.41 \mathrm{MGy})$, measured $\sim 10$ years after $\gamma$ irradiation. In the case of $\mathrm{g}-\mathrm{As}_{2} \mathrm{~S}_{3}$, the observed longterm radiation-induced optical darkening effect is well comparable with that reported in literature for $\gamma$ irradiated ( 3 MGy accumulated dose) g- $\mathrm{As}_{2} \mathrm{~S}_{3}$ (1.5 mm thick) measured directly after $\gamma$-irradiation [10]. In view of practical applications, this finding demonstrates the possibilities for development of innovative chalcogenide glass based long-term dosimeter systems with stable and controlled parameters. Previous investigations of both these samples with the positron annihilation spectroscopy DBAL method and analysis performed in respect to XRD and Raman data allow also suggestion on the possible general criterion for mechanisms of longterm radiation-induced structural changes in ChGs.

\section{Acknowledgments}

The investigated samples used for measurements were prepared within joint research projects (\#0106U007386 and \#0109U007446c) between DSPU (Drohobych, Ukraine) and SRC “Carat” (Lviv, Ukraine) supported by the MES of Ukraine (\#0106U007385 and \#0109U007445). Support of DAAD (Germany) and MES of Ukraine (projects \#0111U001021 and \#0114U002616) is also gratefully acknowledged.

\section{References}

1. O.I. Shpotyuk, Radiation-induced effects in chalcogenide vitreous semiconductors. Chapter 6 // In: Semiconducting Chalcogenide Glass I: Glass Formation, Structure, and Stimulated Transformations in Chalcogenide Glasses: Semiconductors and Semimetals, Vol. 78 
(R. Fairman and B. Ushkov, eds.). Elsevier Academic Press, 2004, p. 215-260.

2. T.S. Kavetskyy, A.L. Stepanov, Effects of gammairradiation and ion implantation in chalcogenide glasses. Chapter 14 // In: Glass Nanocomposites: Synthesis, Properties and Applications (B. Karmakar, K. Rademann, A.L. Stepanov, eds.). Elsevier Academic Press, 2016, p. 341-358.

3. O.I. Shpotyuk, E.R. Skordeva, R.Ya. Golovchak, V.D. Pamukchieva, A.P. Kovalskii, Radiationstimulated changes in transmission of chalcogenide glasses of $\mathrm{As}_{2} \mathrm{~S}_{3}-\mathrm{Ge}_{2} \mathrm{~S}_{3} / / \mathrm{J}$. Appl. Spectr. 66(5), p. 749-753 (1999).

4. O.I. Shpotyuk, T.S. Kavetskyy, A.P. Kovalskiy, R.V. Lutziv, V. Pamukchieva, Radiation-induced changes in optical transmission in vitreous semiconductors of the $\mathrm{Ge}_{x} \mathrm{Sb}_{40-x} \mathrm{~S}_{60}$ system // Ukr. J. Phys. 46(4), p. 495-498 (2001).

5. O. Shpotyuk, S.A. Kozyukhin, M. Shpotyuk, A. Ingram, R. Szatanik, Positronics of radiationinduced effects in chalcogenide glassy semiconductors // Semiconductors, 49(3), p. 298304 (2015).

6. A. Feltz, Amorphous and Vitreous Inorganic Solids. Moscow, Mir, 1986 (in Russian).

7. T. Kavetskyy, I. Kaban, O. Shpotyuk, W. Hoyer, $\mathrm{V}$. Tsmots, On the structural-optical correlations in radiation-modified chalcogenide glasses // J. Phys.: Conf. Ser. 289, p. 012007(1-6) (2011).

8. T. Kavetskyy, O. Shpotyuk, I. Kaban, W. Hoyer, Atomic- and void-species nanostructures in chalcogenide glasses modified by high-energy $\gamma$ irradiation // J. Optoelectron. Adv. Mater. 9(10), p. 3247-3252 (2007).

9. T.S. Kavetskyy, Radiation-induced optical darkening and oxidation effects in $\mathrm{As}_{2} \mathrm{~S}_{3}$ glass // Semiconductor Physics, Quantum Electronics \& Optoelectronics, 17(3), p. 308-312 (2014).

10. M.V. Shpotyuk, M.M. Vakiv, O.I. Shpotyuk, S.B. Ubizskii, On the origin of radiation-induced metastability in vitreous chalcogenide semiconductors: The role of intrinsic and impurityrelated destruction-polymerization transformations // Semiconductor Physics, Quantum Electronics \& Optoelectronics, 18(1), p. 90-96 (2015).

11. T. Kavetskyy, V. Tsmots, O. Šauša, A.L. Stepanov, Structural modification of chalcogenide glasses by gamma-irradiation studied with DBAL technique // Phys. Status Solidi C, 9(12), p. 2420-2423 (2012).
12. T.S. Kavetskyy, V.M. Tsmots, A.L. Stepanov, Radiation/annealing-induced structural changes in $\mathrm{Ge}_{x} \mathrm{As}_{40-x} \mathrm{~S}_{60}$ glasses as revealed from high-energy synchrotron X-ray diffraction measurements // Semiconductor Physics, Quantum Electronics \& Optoelectronics, 15(4), p. 310-320 (2012).

13. T.S. Kavetskyy, Radiation-induced structural changes in chalcogenide glasses as revealed from Raman spectroscopy measurements // Semiconductor Physics, Quantum Electronics \& Optoelectronics, 16(1), p. 27-36 (2013).

14. T.S. Kavetskyy, V.M. Tsmots, O. Šauša, A.L. Stepanov, On the application of methods of positron annihilation spectroscopy for studying radiation-stimulated processes in chalcogenide glassy semiconductors // Semiconductors, 48(1), p. 9-12 (2014).

15. A. Kovalskiy, H. Jain, A.C. Miller, R.Ya. Golovchak, O.I. Shpotyuk, A study of reversible $\gamma$-induced structural transformations in vitreous $\mathrm{Ge}_{23.5} \mathrm{Sb}_{11.8} \mathrm{~S}_{64.7}$ by high-resolution $\mathrm{X}$-ray photoelectron spectroscopy // J. Phys. Chem. B, 110, p. 22930-22934 (2006).

16. T. Kavetskyy, O. Shpotyuk, I. Kaban, W. Hoyer, Radiation-modified structure of $\mathrm{Ge}_{25} \mathrm{Sb}_{15} \mathrm{~S}_{60}$ and $\mathrm{Ge}_{35} \mathrm{Sb}_{5} \mathrm{~S}_{60}$ glasses // J. Chem. Phys. 128(24), p. 244514-1-244514-8 (2008).

17. T. Kavetskyy, O. Shpotyuk, V. Balitska, G. Dovbeshko, I. Blonskyy, I. Kaban, W. Hoyer, M. Iovu, A. Andriesh, Vibrational and structural properties of unmodified and radiation-modified chalcogenide glasses for advanced optical applications // Proc. SPIE, 7142, p. 71420B-171420B-8 (2008).

18. S.R. Elliott, Medium-range structural order in covalent amorphous solids // Nature, 354, p. 445452 (1991).

19. A.P. Sokolov, A. Kisliuk, M. Soltwisch, D. Quitmann, Medium-range order in glasses: Comparison of Raman and diffraction measurements // Phys. Rev. Lett. 69(10), p. 15401543 (1992).

20. L. Börjesson, A.K. Hassan, J. Swenson, L.M. Torell, A. Fontana, Is there a correlation between the first sharp diffraction peak and the low frequency vibrational behavior of glasses? // Phys. Rev. Lett. 70(9), p. 1275-1278 (1993). 